\title{
WEYL TYPE THEOREMS FOR FUNCTIONS OF OPERATORS
}

\author{
SEN ZHU \\ Department of Mathematics, Jilin University, Changchun 130012, P.R. China \\ e-mail: zhusen@jlu.edu.cn \\ CHUN GUANG LI \\ Institute of Mathematics, Jilin University, Changchun 130012, P.R. China \\ e-mail: licg09@mails.jlu.edu.cn \\ and TING TING ZHOU \\ Institute of Mathematics, Jilin University, Changchun 130012, P.R. China \\ e-mail: zhoutt09@mails.jlu.edu.cn
}

(Received 5 December 2010; revised 19 August 2011; accepted 30 October 2011; first published online 30 March 2012)

\begin{abstract}
A-Weyl's theorem and property $(\omega)$, as two variations of Weyl's theorem, were introduced by Rakočević. In this paper, we study a-Weyl's theorem and property $(\omega)$ for functions of bounded linear operators. A necessary and sufficient condition is given for an operator $T$ to satisfy that $f(T)$ obeys a-Weyl's theorem (property $(\omega)$ ) for all $f \in \operatorname{Hol}(\sigma(T))$. Also we investigate the small-compact perturbations of operators satisfying a-Weyl's theorem (property $(\omega)$ ) in the setting of separable Hilbert spaces.
\end{abstract}

2000 Mathematics Subject Classification. Primary 47A53, 47A60; Secondary $47 \mathrm{~A} 10$.

1. Introduction. This paper is a continuation of a previous paper of the authors and Feng [17], where the stability of Weyl's theorem under holomorphic functional calculus is studied. A-Weyl's theorem and property $(\omega)$ as two variations of Weyl's theorem, which have been recently studied in $[3,4,5]$, were introduced by Rakočević $[\mathbf{2 1}, \mathbf{2 2}]$. The purpose of this paper is to investigate a-Weyl's theorem and property $(\omega)$ for functions of operators on Banach spaces. A necessary and sufficient condition is given for an operator $T$ to satisfy that $f(T)$ obeys a-Weyl's theorem (property $(\omega)$ ) for each function $f$ analytic on some neighbourhood of $\sigma(T)$.

We first give some notations and terminologies. Throughout this paper, $\mathbb{C}$ and $\mathbb{N}$ denote the set of complex numbers and the set of natural numbers respectively. $\mathcal{X}$ will always denote a complex infinite dimensional Banach space. We let $\mathcal{B}(\mathcal{X})$ denote the algebra of all bounded linear operators on $\mathcal{X}$, and let $\mathcal{K}(\mathcal{X})$ denote the ideal of compact operators in $\mathcal{B}(\mathcal{X})$.

Let $T \in \mathcal{B}(\mathcal{X})$. We denote by $\sigma(T)$ and $\sigma_{p}(T)$ the spectrum of $T$ and the point spectrum of $T$ respectively. Denote by $n(T)$ and $\mathcal{R}(T)$ the kernel of $T$ and the range

This research is supported by NNSF of China (10971079, 11026038, 11101177), the Basic Research Foundation of Jilin University (201001001, 201103194) and the Young Fund of Department of Mathematics at Jilin University. 
of $T$ respectively. $T$ is called a semi-Fredholm operator, if $\mathcal{R}(T)$ is closed and either nul $T$ or nul $T^{*}$ is finite, where nul $T:=\operatorname{dim} n(T)$ and nul $T^{*}:=\operatorname{dim} n\left(T^{*}\right)$; in this case, ind $T:=$ nul $T-$ nul $T^{*}$ is called the index of $T$. In particular, if $-\infty<$ ind $T<\infty$, then $T$ is called a Fredholm operator. It is well known that if $T$ is semi-Fredholm and $K \in \mathcal{K}(\mathcal{X})$, then $T+K$ is also semi-Fredholm and ind $(T+K)=$ ind $T . T$ is called a Weyl operator if it is Fredholm of index 0.

The Wolf spectrum $\sigma_{\text {lre }}(T)$, the essential spectrum $\sigma_{e}(T)$ and the Weyl spectrum $\sigma_{w}(T)$ of $T$ are defined as:

$$
\begin{gathered}
\sigma_{\text {lre }}(T):=\{\lambda \in \mathbb{C}: T-\lambda \text { is not semi-Fredholm }\}, \\
\sigma_{e}(T):=\{\lambda \in \mathbb{C}: T-\lambda \text { is not Fredholm }\}
\end{gathered}
$$

and

$$
\sigma_{w}(T):=\{\lambda \in \mathbb{C}: T-\lambda \text { is not Weyl }\},
$$

respectively. $\rho_{s-F}(T):=\mathbb{C} \backslash \sigma_{\text {lre }}(T)$ is called the semi-Fredholm domain of $T$. The approximate point spectrum $\sigma_{a}(T)$ and the essential approximate point spectrum $\sigma_{e a}(T)$ of $T$ are defined as:

$$
\sigma_{a}(T)=\{\lambda \in \mathbb{C}: \lambda-T \text { is not bounded below }\}
$$

and

$$
\sigma_{e a}(T)=\bigcap_{K \in \mathcal{K}(\mathcal{X})} \sigma_{a}(T+K),
$$

respectively. The set $\sigma_{e a}(T)$ has been introduced in [20] and studied in [20, 21, 23]. It is easy to see that

$$
\sigma_{e a}(T)=\sigma_{\text {lre }}(T) \cup\left\{\lambda \in \rho_{s-F}(T): \text { ind }(T-\lambda)>0\right\} .
$$

Given a subset $\sigma$ of $\mathbb{C}$, denote by iso $\sigma$ and int $\sigma$ the set of all isolated points of $\sigma$ and the interior of $\sigma$ respectively. We denote

$$
\pi_{00}(T):=\{\lambda \in \text { iso } \sigma(T): 0<\operatorname{nul}(\lambda-T)<\infty\}
$$

and

$$
\pi_{00}^{a}(T):=\left\{\lambda \in \text { iso } \sigma_{a}(T): 0<\operatorname{nul}(\lambda-T)<\infty\right\} .
$$

Following Coburn [9], we say that Weyl's theorem holds for $T \in \mathcal{B}(\mathcal{X})$, denoted by $T \in(\mathrm{W})$, if $\sigma(T) \backslash \sigma_{w}(T)=\pi_{00}(T)$. Today, Weyl's theorem has been extended to various operators acting on both Hilbert spaces and Banach spaces, and there has been a lot of work (see, for example, $[\mathbf{6}, \mathbf{8}, \mathbf{1 1}, \mathbf{1 2}, \mathbf{1 3}, \mathbf{1 4}])$. We say that a-Weyl's theorem holds for $T \in \mathcal{B}(\mathcal{X})$, denoted by $T \in(\mathrm{a}-\mathrm{W})$, if $\sigma_{a}(T) \backslash \sigma_{e a}(T)=\pi_{00}^{a}(T)$. A-Weyl's theorem has been introduced and studied in [21]. $T$ is said to have property $(\omega)$, denoted by $T \in(\omega)$, if $\sigma_{a}(T) \backslash \sigma_{e a}(T)=\pi_{00}(T)$. It is well known that both $T \in(\mathrm{a}-\mathrm{W})$ and $T \in(\omega)$ imply that $T \in(\mathrm{W})$ (see [2]). 
Let $\operatorname{Hol}(\sigma(T))$ denote the set of all functions $f$ which are analytic on some neighbourhood of $\sigma(T)$ (the neighbourhood depends on $f$ ) for given $f \in \operatorname{Hol}(\sigma(T)$ ), $f(T)$ denotes the holomorphic functional calculus of $T$ with respect to $f$.

Let $T \in \mathcal{B}(\mathcal{X})$. If $\sigma$ is a clopen subset of $\sigma(T)$, then there exists an analytic Cauchy domain $\Omega$ such that $\sigma \subseteq \Omega$ and $[\sigma(T) \backslash \sigma] \cap \bar{\Omega}=\emptyset$. We let $E(\sigma ; T)$ denote the Riesz idempotent of $T$ corresponding to $\sigma$, that is,

$$
E(\sigma ; T)=\frac{1}{2 \pi i} \int_{\Gamma}(\lambda-T)^{-1} \mathrm{~d} \lambda,
$$

where $\Gamma=\partial \Omega$ is positively oriented with respect to $\Omega$ in the sense of complex variable theory. In this case, we denote $\mathcal{X}(\sigma ; T)=\mathcal{R}(E(\sigma ; T))$. If $\lambda \in$ iso $\sigma(T)$, then $\{\lambda\}$ is a clopen subset of $\sigma(T)$ and we simply write $\mathcal{X}(\lambda ; T)$ instead of $\mathcal{X}(\{\lambda\} ; T)$; if, in addition, $\operatorname{dim} \mathcal{X}(\lambda ; T)<\infty$, then $\lambda$ is called a normal eigenvalue of $T$. A normal eigenvalue of $T$ is also called a Riesz point of $T$ (see [7]). The set of all normal eigenvalues of $T$ will be denoted by $\sigma_{0}(T)$.

We denote

$$
\begin{gathered}
\rho_{s-F}^{0}(T):=\{\lambda \in \mathbb{C}: T-\lambda \text { is Weyl }\}, \\
\rho_{s-F}^{+}(T):=\left\{\lambda \in \rho_{s-F}(T): \text { ind }(T-\lambda)>0\right\}
\end{gathered}
$$

and

$$
\rho_{s-F}^{-}(T):=\left\{\lambda \in \rho_{s-F}(T): \text { ind }(T-\lambda)<0\right\} .
$$

Obviously, $\rho_{s-F}(T)=\rho_{s-F}^{-}(T) \cup \rho_{s-F}^{0}(T) \cup \rho_{s-F}^{+}(T)$ and $\rho_{s-F}^{0}(T)=\mathbb{C} \backslash \sigma_{w}(T)$.

Now, we can list the main results of this paper.

MAIN THEOREM 1.1. Let $T \in \mathcal{B}(\mathcal{X})$. Then, $f(T) \in(\mathrm{a}-\mathrm{W})$ for all $f \in \operatorname{Hol}(\sigma(T))$ if and only if the following conditions hold.

(i) $T \in(\mathrm{a}-\mathrm{W})$.

(ii) If $\rho_{s-F}^{-}(T) \neq \emptyset$, then there exists no $\lambda \in \rho_{s-F}(T)$ such that $0<$ ind $(T-\lambda)<\infty$.

(iii) If $\sigma_{p}(T) \cap\left[\rho_{s-F}^{-}(T) \cup \rho_{s-F}^{0}(T)\right] \neq \emptyset$, then iso $\sigma_{a}(T) \subseteq \sigma_{p}(T)$.

It is worth mentioning that Weyl type theorems are closely related to some basic concepts in local spectral theory (see [1]). Oudghiri [18] related Weyl's theorem to the single-valued extension property in local spectral theory. In [2], Aiena gave some sufficient conditions for an operator $T$ to satisfy $f(T) \in(\mathrm{a}-\mathrm{W})$ for all $f \in \operatorname{Hol}(\sigma(T))$ in terms of certain glocal spectral subspaces.

MAIN THEOREM 1.2. Let $T \in \mathcal{B}(\mathcal{X})$. Then, $f(T) \in(\omega)$ for all $f \in \operatorname{Hol}(\sigma(T))$ if and only if the following conditions hold.

(i) $T \in(\omega)$.

(ii) If $\rho_{s-F}^{-}(T) \neq \varnothing$, then $\sigma_{0}(T)=\emptyset$ and there exists no $\lambda \in \rho_{s-F}(T)$ such that $0<$ ind $(T-\lambda)<\infty$.

(iii) If $\sigma_{0}(T) \neq \emptyset$, then iso $\sigma(T) \subseteq \sigma_{p}(T)$.

If $\mathcal{X}$ is a complex separable Hilbert space and $\operatorname{dim} \mathcal{X}=\infty$, then it is proved in [17] that any operator $T \in \mathcal{B}(\mathcal{X})$ has an arbitrarily small compact perturbation satisfying Weyl's theorem. Since $A \in(\omega)$ implies that $A$ satisfies Weyl's theorem, the following theorem strengthens the above result. 
MAIN THEOREM 1.3. Let $\mathcal{X}$ be a complex separable infinite dimensional Hilbert space. Then, given $T \in \mathcal{B}(\mathcal{X})$ and $\varepsilon>0$, there exists $K \in \mathcal{K}(\mathcal{X})$ with $\|K\|<\varepsilon$ such that $T+K \in(\omega)$ and $T+K \in(\mathrm{a}-\mathrm{W})$.

The rest part of this paper is organized as follows. In Section 2, we shall make some preparations for the proofs of main theorems. Section 3 is devoted to the proof of Main Theorem 1.1. The proofs of main theorem 1.2/1.3 shall be provided respectively in Section 4 and Section 5.

2. Preparations. In this section, we give some useful lemmas.

LeMma 2.1 ([19], Theorem 2.10). Let $T \in \mathcal{B}(\mathcal{X})$ and suppose that $\sigma(T)=$ $\sigma_{1} \cup \sigma_{2}$, where $\sigma_{i}(i=1,2)$ are clopen subsets of $\sigma(T)$ and $\sigma_{1} \cap \sigma_{2}=\emptyset$. Then, $\mathcal{X}\left(\sigma_{1} ; T\right)+\mathcal{X}\left(\sigma_{2} ; T\right)=\mathcal{X}, \mathcal{X}\left(\sigma_{1} ; T\right) \cap \mathcal{X}\left(\sigma_{2} ; T\right)=\{0\}$ and $T$ admits the following matrix representation

$$
T=\left[\begin{array}{cc}
T_{1} & 0 \\
0 & T_{2}
\end{array}\right] \mathcal{X}\left(\sigma_{1} ; T\right),
$$

where $\sigma\left(T_{i}\right)=\sigma_{i}(i=1,2)$.

Using [15, Corollary 3.22] and the above lemma, we can obtain the following lemma whose proof is left to the reader.

COROLlaRY 2.2. Let $\mathcal{X}$ be a complex separable Hilbert space and $T \in \mathcal{B}(\mathcal{X})$. Suppose that $\sigma$ is a clopen subset of $\sigma(T)$. Then

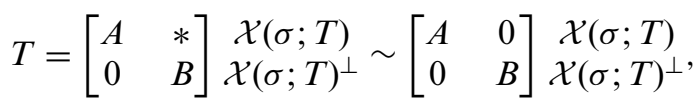

where $\sigma(A)=\sigma$ and $\sigma(B)=\sigma(T) \backslash \sigma$.

In this paper, if $S, T \in \mathcal{B}(\mathcal{X})$, then we let $S \sim T$ denote that $S$ and $T$ are similar.

Lemma 2.3 ([10], chapter XI, proposition 6.9). Let $T \in \mathcal{B}(\mathcal{X})$ and $\lambda_{0} \in$ iso $\sigma(T)$. Then, the following statements are equivalent.

(i) $\lambda_{0} \in \sigma_{0}(T)$.

(ii) $\lambda_{0} \in \rho_{s-F}^{0}(T)$.

(iii) $\lambda_{0} \in \rho_{s-F}(T)$.

Note that an operator $T \in \mathcal{B}(\mathcal{X})$ is bounded below if and only if nul $T=0$ and $\mathcal{R}(T)$ is closed, then, by the continuity of the index function ind $(\cdot)$, the following lemma is clear.

LemmA 2.4. Let $T \in \mathcal{B}(\mathcal{X})$ and $\lambda_{0} \in$ iso $\sigma_{a}(T)$. If $\lambda_{0} \in \rho_{s-F}(T)$, then $0<\operatorname{nul}(T-$ $\left.\lambda_{0}\right)<\infty$ and ind $\left(T-\lambda_{0}\right) \leq 0$.

The proof of the following lemma is simple and we omit it.

Lemma 2.5. Let $T \in \mathcal{B}(\mathcal{X})$ and $f \in \operatorname{Hol}(\sigma(T))$. Then, $f\left(\sigma_{a}(T)\right) \subseteq \sigma_{a}(f(T))$ and $f\left(\sigma_{p}(T)\right) \subseteq \sigma_{p}(f(T))$.

In this paper, we denote by card $\sigma$ the cardinality of a subset $\sigma$ of $\mathbb{C}$. If $\lambda \in \mathbb{C}$ and $\delta>0$, then we denote $B_{\delta}(\lambda)=\{z \in \mathbb{C}:|z-\lambda|<\delta\}$. 
LEMMA 2.6. Let $T \in \mathcal{B}(\mathcal{X})$ and $f \in \operatorname{Hol}(\sigma(T))$. If $0 \in \sigma(f(T))$ and nul $f(T)<\infty$, then $1 \leq$ card $\{\lambda \in \sigma(T): f(\lambda)=0\}<\infty$; if, in addition, $0 \in$ iso $\sigma_{a}(f(T))$, then for each $\mu \in\{\lambda \in \sigma(T): f(\lambda)=0\}$, there exists $\delta>0$ such that $B_{\delta}(\mu) \backslash\{\mu\} \subseteq \mathbb{C} \backslash \sigma_{a}(T)$.

Proof. It is obvious that $\{\lambda \in \sigma(T): f(\lambda)=0\} \neq \emptyset$. If $\{\lambda \in \sigma(T): f(\lambda)=0\}$ is an infinite subset of $\sigma(T)$, then we can choose a limit point $\lambda_{0}$ of $\{\lambda \in \sigma(T): f(\lambda)=0\}$. Without loss of generality, we assume that $f$ is analytic on a neighbourhood $\Omega$ of $\sigma(T)$. Then, there is a component $\Omega_{1}$ of $\Omega$ such that $\lambda_{0} \in \Omega_{1}$ and $f \equiv 0$ on $\Omega_{1}$. Set $\sigma_{1}=\sigma(T) \cap \Omega_{1}$ and $\sigma_{2}=\sigma(T) \backslash \sigma_{1}$. Then, $\sigma_{i}(i=1,2)$ are clopen subsets of $\sigma(T)$ and $\sigma_{1} \neq \emptyset$.

By Lemma 2.1, $T$ can be written as

$$
T=\left[\begin{array}{cc}
T_{1} & 0 \\
0 & T_{2}
\end{array}\right] \mathcal{X}\left(\sigma_{1} ; T\right),
$$

where $\sigma\left(T_{i}\right)=\sigma_{i}(i=1,2)$. Hence, $f\left(T_{1}\right)=0$. Since $\lambda_{0}$ is a limit point of $\{\lambda \in \sigma(T)$ : $f(\lambda)=0\}, \lambda_{0} \in \sigma_{1}$ and $\sigma_{1}$ is a clopen subset of $\sigma(T)$, it is easy to see that card $\left\{\lambda \in \sigma_{1}\right.$ : $f(\lambda)=0\}=\infty$ and $\operatorname{dim} \mathcal{X}\left(\sigma_{1} ; T\right)=\infty$. Then, we have

$$
f(T)=\left[\begin{array}{cc}
f\left(T_{1}\right) & 0 \\
0 & f\left(T_{2}\right)
\end{array}\right] \begin{aligned}
& \mathcal{X}\left(\sigma_{1} ; T\right) \\
& \mathcal{X}\left(\sigma_{2} ; T\right)
\end{aligned}=\left[\begin{array}{cc}
0 & 0 \\
0 & f\left(T_{2}\right)
\end{array}\right] \begin{aligned}
& \mathcal{X}\left(\sigma_{1} ; T\right) \\
& \mathcal{X}\left(\sigma_{2} ; T\right)
\end{aligned}
$$

It follows immediately that nul $f(T) \geq \operatorname{dim} \mathcal{X}\left(\sigma_{1} ; T\right)=\infty$, a contradiction. Thus, we have proved that card $\{\lambda \in \sigma(T): f(\lambda)=0\}<\infty$.

Now we assume that $0 \in$ iso $\sigma_{a}(f(T))$ and $\mu \in \sigma(T)$ satisfies that $f(u)=0$. We shall prove that there exists $\delta>0$ such that $B_{\delta}(\mu) \backslash\{\mu\} \subseteq \mathbb{C} \backslash \sigma_{a}(T)$. If not, then we can choose $\left\{\mu_{n}\right\}_{n=1}^{\infty} \subseteq\left[\sigma_{a}(T) \backslash\{\mu\}\right]$ such that $\mu_{n} \rightarrow \mu$. By Lemma 2.5, $f\left(\mu_{n}\right) \in \sigma_{a}(f(T))$ for all $n$ and $f\left(\mu_{n}\right) \rightarrow f(\mu)=0$. Since card $\{\lambda \in \sigma(T): f(\lambda)=0\}<\infty$, we may assume that $f\left(\mu_{n}\right) \neq 0$ for all $n \geq 1$. Thus, we obtain $0 \notin$ iso $\sigma_{a}(f(T))$, a contradiction.

Using a similar argument as in the proof of Lemma 2.6, one can obtain the following result.

Corollary 2.7. Let $T \in \mathcal{B}(\mathcal{X})$ and $f \in \operatorname{Hol}(\sigma(T))$. If $0 \in$ iso $\sigma(f(T))$ and nul $f(T)<\infty$, then $1 \leq$ card $\{\lambda \in \sigma(T): f(\lambda)=0\}<\infty$ and $\{\lambda \in \sigma(T): f(\lambda)=0\} \subseteq$ iso $\sigma(T)$.

Lemma 2.8 ([17], Lemma 2.7). Let $T \in \mathcal{B}(\mathcal{X})$ and $f \in \operatorname{Hol}(\sigma(T))$. If $0 \in \sigma(f(T))$ and nul $f(T)<\infty$, then, there exists $g \in \operatorname{Hol}(\sigma(T))$ such that $f(T)=g(T)$ and

$$
g(\lambda)=\left(\lambda-\lambda_{1}\right)^{k_{1}}\left(\lambda-\lambda_{2}\right)^{k_{2}} \cdots\left(\lambda-\lambda_{n}\right)^{k_{n}} g_{0}(\lambda),
$$

where $\lambda_{i} \in \sigma(T)(1 \leq i \leq n), g_{0} \in \operatorname{Hol}(\sigma(T))$ and $g_{0}(\lambda) \neq 0$ for all $\lambda \in \sigma(T)$.

Lemma 2.9. Let $T \in \mathcal{B}(\mathcal{X})$. Suppose that $\lambda_{i} \in \sigma(T)(1 \leq i \leq n)$ and $f(\lambda)=(\lambda-$ $\left.\lambda_{1}\right)^{k_{1}} \cdots\left(\lambda-\lambda_{n}\right)^{k_{n}} g(\lambda)$, where $g \in \operatorname{Hol}(\sigma(T))$ and $g(\lambda) \neq 0$ for all $\lambda \in \sigma(T)$. For each $i$, there exists a $\delta_{i}>0$ such that $\left[B_{\delta_{i}}\left(\lambda_{i}\right) \backslash\left\{\lambda_{i}\right\}\right] \subseteq\left[\mathbb{C} \backslash \sigma_{a}(T)\right]$. Then, there exists $\delta>0$ such that $\left[B_{\delta}(0) \backslash\{0\}\right] \subseteq\left[\mathbb{C} \backslash \sigma_{a}(f(T))\right]$.

Proof. Without loss of generality, we assume that $\left\{B_{\delta_{i}}\left(\lambda_{i}\right)\right\}_{i=1}^{n}$ are pairwise disjoint and $g$ is well defined on $\cup_{i=1}^{n} \overline{B_{\delta_{i}}\left(\lambda_{i}\right)}$. Set $\delta_{0}=\min \{|g(\lambda)|: \lambda \in \sigma(T)\}$ and $\delta=\frac{\delta_{0}}{2} \cdot \Pi_{i=1}^{n} \delta_{i}^{k_{i}}$. Obviously, $\delta>0$ and $0 \in \sigma(f(T))$. 
Arbitrarily choose a $\lambda_{0} \in B_{\delta}(0), \lambda_{0} \neq 0$. We shall prove that $\lambda_{0}-f(T)$ is bounded below. Without loss of generality, we may assume that $\lambda_{0} \in \sigma(f(T))$.

ClaIM. If $\mu \in \sigma(T)$ and $f(\mu)=\lambda_{0}$, then $\mu \in \cup_{i=1}^{n} B_{\delta_{i}}\left(\lambda_{i}\right)$.

In fact, if not, then $\left|\mu-\lambda_{i}\right| \geq \delta_{i}$ for all $i$. Thus,

$$
\delta>\left|\lambda_{0}\right|=|f(\mu)| \geq \delta_{0} \cdot \Pi_{i=1}^{n}\left|\mu-\lambda_{i}\right|^{k_{i}} \geq \delta_{0} \cdot \Pi_{i=1}^{n} \delta_{i}^{k_{i}}>\delta,
$$

a contradiction.

Since $\left|\lambda_{0}\right|<|f(\lambda)|$ on $\cup_{i=1}^{n} \partial B_{\delta_{i}}\left(\lambda_{i}\right)$, by Rouché's theorem, we deduce that $f(\lambda)$ and $f(\lambda)-\lambda_{0}$ have the same number of zeros in $\cup_{i=1}^{n} B_{\delta_{i}}\left(\lambda_{i}\right)$, where each zero is counted as many times as its multiplicity. Hence, we may assume that

$$
f(\lambda)-\lambda_{0}=\left(\lambda-\mu_{1}\right) \cdots\left(\lambda-\mu_{m}\right) f_{0}(\lambda)
$$

where $f_{0} \in \operatorname{Hol}(\sigma(T))$ and $f_{0}(\lambda) \neq 0$ for all $\lambda \in \sigma(T)$. Here, $\mu_{i}(1 \leq i \leq m)$ may repeat according to multiplicity. Then,

$$
f(T)-\lambda_{0}=\left(T-\mu_{1}\right)\left(T-\mu_{2}\right) \cdots\left(T-\mu_{m}\right) f_{0}(T),
$$

where $f_{0}(T)$ is invertible.

Note that $\mu_{i} \neq \lambda_{j}$ for all $i$ and $j$ (otherwise $\lambda_{0}=f\left(\mu_{i}\right)=f\left(\lambda_{j}\right)=0$, a contradiction). Then, by our claim, we have

$$
\left\{\mu_{i}: 1 \leq i \leq m\right\} \subseteq \cup_{j=1}^{n}\left[B_{\delta_{j}}\left(\lambda_{j}\right) \backslash\left\{\lambda_{j}\right\}\right] .
$$

Therefore, $T-\mu_{i}$ is bounded below for all $i$ and hence $f(T)-\lambda_{0}$ is bounded below.

Using a similar argument as in the proof of Lemma 2.9, one can obtain the following result.

COROLlary 2.10. Let $T \in \mathcal{B}(\mathcal{X})$ and suppose that $\lambda_{i} \in$ iso $\sigma(T)(1 \leq i \leq n)$. Iff $(\lambda)=$ $\left(\lambda-\lambda_{1}\right)^{k_{1}} \cdots\left(\lambda-\lambda_{n}\right)^{k_{n}} g(\lambda)$, where $g \in \operatorname{Hol}(\sigma(T))$ and $g(\lambda) \neq 0$ for all $\lambda \in \sigma(T)$, then $0 \in$ iso $\sigma(f(T))$.

\section{Proof of Main Theorem 1.1.}

LemMa 3.1. Let $T \in \mathcal{B}(\mathcal{X})$. Then,

$$
\sigma_{a}(T) \backslash \sigma_{e a}(T)=\left[\rho_{s-F}^{-}(T) \cup \rho_{s-F}^{0}(T)\right] \cap \sigma_{p}(T) .
$$

Hence,

$$
T \in(\mathrm{a}-\mathrm{W}) \Longleftrightarrow\left[\rho_{s-F}^{-}(T) \cup \rho_{s-F}^{0}(T)\right] \cap \sigma_{p}(T)=\pi_{00}^{a}(T)
$$

and

$$
T \in(\omega) \Longleftrightarrow\left[\rho_{s-F}^{-}(T) \cup \rho_{s-F}^{0}(T)\right] \cap \sigma_{p}(T)=\pi_{00}(T) .
$$

Proof. Obviously, we need only prove that

$$
\sigma_{a}(T) \backslash \sigma_{e a}(T)=\left[\rho_{s-F}^{-}(T) \cup \rho_{s-F}^{0}(T)\right] \cap \sigma_{p}(T) .
$$


The inclusion relation " $\supseteq$ " is obvious. We only prove that the inclusion relation " $\subseteq$ " holds. $\lambda_{0} \in\left[\sigma_{a}(T) \backslash \sigma_{e a}(T)\right]$ implies that $\lambda_{0} \notin \sigma_{e a}(T)$, that is, there exists $K \in \mathcal{K}(\mathcal{X})$ such that $T+K-\lambda_{0}$ is bounded below. Hence, ind $\left(T-\lambda_{0}\right)=$ ind $\left(T+K-\lambda_{0}\right) \leq 0$. Note that $\lambda_{0} \in \sigma_{a}(T)$, then nul $\left(T-\lambda_{0}\right)>0$. Thus, we obtain $\lambda_{0} \in\left[\rho_{s-F}^{-}(T) \cup \rho_{s-F}^{0}(T)\right] \cap$ $\sigma_{p}(T)$.

Recall that a set, which is made up only of isolated points, is called a discrete set. The following result provides a necessary and sufficient condition for an operator to satisfy a-Weyl's theorem.

Lemma 3.2. Let $T \in \mathcal{B}(\mathcal{X})$. Then, $T \in(\mathrm{a}-\mathrm{W})$ if and only if the following conditions hold:

(i) $\left[\rho_{s-F}^{0}(T) \cup \rho_{s-F}^{-}(T)\right] \cap \sigma_{p}(T)$ is a discrete set;

(ii) $\pi_{00}^{a}(T) \subseteq \rho_{s-F}(T)$.

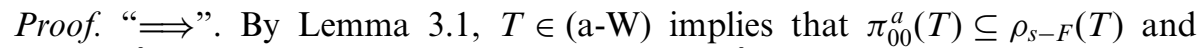
$\left[\rho_{s-F}^{-}(T) \cup \rho_{s-F}^{0}(T)\right] \cap \sigma_{p}(T) \subseteq$ iso $\sigma_{a}(T)$. Hence, $\left[\rho_{s-F}^{0}(T) \cup \rho_{s-F}^{-}(T)\right] \cap \sigma_{p}(T)$ is a discrete set.

“£". By Lemma 2.4, it follows easily from $\pi_{00}^{a}(T) \subseteq \rho_{s-F}(T)$ that $\pi_{00}^{a}(T) \subseteq$ $\left[\rho_{s-F}^{-}(T) \cup \rho_{s-F}^{0}(T)\right] \cap \sigma_{p}(T)$. By Lemma 3.1, it suffices to prove that $\left[\rho_{s-F}^{-}(T) \cup\right.$ $\left.\rho_{s-F}^{0}(T)\right] \cap \sigma_{p}(T) \subseteq \pi_{00}^{a}(T)$.

Arbitrarily choose a $\lambda_{0} \in\left[\rho_{s-F}^{-}(T) \cup \rho_{s-F}^{0}(T)\right] \cap \sigma_{p}(T)$. Then, by condition (i), there exists a $\delta_{1}>0$ such that nul $(\lambda-T)=0$ for all $\lambda \in B_{\delta_{1}}\left(\lambda_{0}\right) \backslash\left\{\lambda_{0}\right\}$. Note that $\lambda_{0} \in \rho_{s-F}^{-}(T) \cup \rho_{s-F}^{0}(T)$ and $\rho_{s-F}^{-}(T) \cup \rho_{s-F}^{0}(T)$ is open, then there exists $\delta_{2}>0$ such that $B_{\delta_{2}}\left(\lambda_{0}\right) \subseteq \rho_{s-F}^{-}(T) \cup \rho_{s-F}^{0}(T)$. Set $\delta=\min \left\{\delta_{1}, \delta_{2}\right\}$. Then, it is easy to see that $\lambda-T$ is bounded below for all $\lambda \in B_{\delta}\left(\lambda_{0}\right) \backslash\left\{\lambda_{0}\right\}$. Then, it follows from $\lambda_{0} \in\left[\rho_{s-F}^{-}(T) \cup\right.$ $\left.\rho_{s-F}^{0}(T)\right] \cap \sigma_{p}(T)$ that $\lambda_{0} \in \pi_{00}^{a}(T)$.

Corollary 3.3. Let $T \in \mathcal{B}(\mathcal{X})$ and suppose that $T \in(\mathrm{a}-\mathrm{W})$. If $\lambda \in \rho_{s-F}(T)$ and ind $(\lambda-T) \leq 0$, then either $\lambda \notin \sigma_{a}(T)$ or $\lambda \in \pi_{00}^{a}(T)$.

Now we are going to give the proof of Main Theorem 1.1.

Proof of Main Theorem 1.1 “”". Assume that $f(T) \in(\mathrm{a}-\mathrm{W})$ for all $f \in$ $\operatorname{Hol}(\sigma(T))$.

(i) Set $f_{1}(\lambda)=\lambda$. Then, evidently, $T=f_{1}(T) \in(\mathrm{a}-\mathrm{W})$.

(ii) If (ii) does not hold, then we can choose $\lambda_{1}, \lambda_{2} \in \mathbb{C}$ such that $0<$ ind ( $T-$ $\left.\lambda_{1}\right)<\infty$ and $-\infty \leq$ ind $\left(T-\lambda_{2}\right)<0$. Obviously, we can choose $k \in \mathbb{N}$ such that ind $\left(T-\lambda_{1}\right)+k \cdot$ ind $\left(T-\lambda_{2}\right)<0$. Define $f_{2}(z)=\left(z-\lambda_{1}\right)\left(z-\lambda_{2}\right)^{k}$. Then, $f_{2}(T)$ is semi-Fredholm and

$$
\text { ind } f_{2}(T)=\text { ind }\left(T-\lambda_{1}\right)+k \cdot \text { ind }\left(T-\lambda_{2}\right)<0 \text {, }
$$

that is, $0 \in \rho_{s-F}^{-}\left(f_{2}(T)\right)$.

ind $\left(T-\lambda_{1}\right)>0$ implies that there exists $\delta>0$ such that ind $(T-\mu)>0$ for all $\mu \in B_{\delta}\left(\lambda_{1}\right)$. Then, nul $(T-\mu) \geq$ ind $(T-\mu)>0$ for all $\mu \in B_{\delta}\left(\lambda_{1}\right)$. Hence, we have $B_{\delta}\left(\lambda_{1}\right) \subseteq \sigma_{p}(T)$. By Lemma 2.5, it follows that $f_{2}\left(B_{\delta}\left(\lambda_{1}\right)\right) \subseteq \sigma_{p}\left(f_{2}(T)\right)$.

Evidently $f_{2}$ is an open mapping, then $f_{2}\left(B_{\delta}\left(\lambda_{1}\right)\right)$ is an open neighbourhood of 0 . Note that $0 \in \rho_{s-F}^{-}\left(f_{2}(T)\right)$, then, we can choose $\varepsilon>0$ such that $B_{\varepsilon}(0) \subseteq$ $f_{2}\left(B_{\delta}\left(\lambda_{1}\right)\right) \cap \rho_{s-F}^{-}\left(f_{2}(T)\right) \subseteq \sigma_{p}\left(f_{2}(T)\right) \cap \rho_{s-F}^{-}\left(f_{2}(T)\right)$. By Lemma 3.2, we have $f_{2}(T) \notin$ $(\mathrm{a}-\mathrm{W})$, a contradiction. 
(iii) If (iii) does not hold, then we can choose $\lambda_{1} \in \sigma_{p}(T) \cap\left[\rho_{s-F}^{-}(T) \cup \rho_{s-F}^{0}(T)\right]$ and $\lambda_{2} \in$ iso $\sigma_{a}(T)$ such that $\lambda_{2} \notin \sigma_{p}(T)$. By Lemma 3.1, it follows from $T \in(\mathrm{a}-\mathrm{W})$ that $\lambda_{1} \in \pi_{00}^{a}(T)$. It follows from Lemma 2.4 that $\lambda_{2} \in \sigma_{\text {lre }}(T)$.

Define $f_{3}(z)=\left(z-\lambda_{1}\right)\left(z-\lambda_{2}\right)$. Then, $0<\operatorname{nul} f_{3}(T)<\infty$. By Lemma 2.5 and Lemma 2.9, it follows from $\lambda_{1} \in$ iso $\sigma_{a}(T)$ and $\lambda_{2} \in$ iso $\sigma_{a}(T)$ that $0 \in$ iso $\sigma_{a}\left(f_{3}(T)\right)$. Hence, we have $0 \in \pi_{00}^{a}\left(f_{3}(T)\right)$. Since $f_{3}(T) \in(\mathrm{a}-\mathrm{W})$, by Lemma 3.2, $f_{3}(T)$ is semiFredholm and ind $f_{3}(T) \leq 0$. Note that $f_{3}(T)=\left(T-\lambda_{1}\right)\left(T-\lambda_{2}\right)$, then we deduce that $T-\lambda_{2}$ is semi-Fredholm, a contradiction.

"£". Arbitrarily choose an $f \in \operatorname{Hol}(\sigma(T))$. It suffices to prove that $f(T) \in(\mathrm{a}-\mathrm{W})$.

Step 1. $\sigma_{a}(f(T)) \backslash \sigma_{e a}(f(T)) \subseteq \pi_{00}^{a}(f(T))$.

Let $\lambda_{0} \in \sigma_{a}(f(T)) \backslash \sigma_{e a}(f(T))$ be fixed. Then, by Lemma 3.1, $0<\operatorname{nul}\left(f(T)-\lambda_{0}\right)<$ $\infty$ and ind $\left(f(T)-\lambda_{0}\right) \leq 0$. It suffices to prove that $\lambda_{0} \in \pi_{00}^{a}(f(T))$. By Lemma 2.6, we have card $\left\{z \in \sigma(T): f(z)-\lambda_{0}=0\right\}<\infty$. Assume $\left\{\lambda_{i}\right\}_{i=1}^{n}$ is an enumeration of $\left\{z \in \sigma(T): f(z)-\lambda_{0}=0\right\}$. Then, by Lemma 2.8, we may assume that

$$
f(z)-\lambda_{0}=\left(z-\lambda_{1}\right)^{k_{1}} \cdots\left(z-\lambda_{n}\right)^{k_{n}} g(z),
$$

where $g(z) \neq 0$ for all $z \in \sigma(T)$. Hence,

$$
f(T)-\lambda_{0}=\left(T-\lambda_{1}\right)^{k_{1}} \cdots\left(T-\lambda_{n}\right)^{k_{n}} g(T),
$$

where $g(T)$ is invertible.

It follows from ind $\left(f(T)-\lambda_{0}\right) \leq 0$ that $\lambda_{i} \in \rho_{s-F}(T)$ and ind $\left(\lambda_{i}-T\right)<\infty$ for all $i$. Then, $\sum_{i=1}^{n} k_{i} \cdot$ ind $\left(T-\lambda_{i}\right)=$ ind $\left(\lambda_{0}-f(T)\right) \leq 0$. It follows from condition (ii) that ind $\left(T-\lambda_{i}\right) \leq 0$ for $1 \leq i \leq n$. By Corollary 3.3, for each $1 \leq i \leq n$, we have either $\lambda_{i} \notin$ $\sigma_{a}(T)$ or $\lambda_{i} \in$ iso $\sigma_{a}(T)$. Then, by Lemma 2.9 , either $\lambda_{0} \notin \sigma_{a}(f(T))$ or $\lambda_{0} \in$ iso $\sigma_{a}(f(T))$. Since $0<\operatorname{nul}\left(f(T)-\lambda_{0}\right)<\infty$, we can conclude that $\lambda_{0} \in \pi_{00}^{a}(f(T))$.

Step 2. $\pi_{00}^{a}(f(T)) \subseteq\left[\sigma_{a}(f(T)) \backslash \sigma_{e a}(f(T))\right]$.

Arbitrarily choose a $\lambda_{0} \in \pi_{00}^{a}(f(T))$. Then, $0<\operatorname{nul}\left(f(T)-\lambda_{0}\right)<\infty$ and $\lambda_{0} \in$ iso $\sigma_{a}(f(T))$. By Lemma 3.1 and Lemma 2.4, it suffices to prove that $\lambda_{0} \in \rho_{s-F}(f(T))$. Note that nul $\left(f(T)-\lambda_{0}\right)<\infty$, then, by Lemma 2.6 and Lemma 2.8, we may assume that $\left\{\lambda_{i}\right\}_{i=1}^{n}$ is an enumeration of $\left\{\lambda \in \sigma(T): f(\lambda)-\lambda_{0}=0\right\}$ and

$$
f(z)-\lambda_{0}=\left(z-\lambda_{1}\right)^{k_{1}} \cdots\left(z-\lambda_{n}\right)^{k_{n}} g(z)
$$

where $g(z) \neq 0$ for all $z \in \sigma(T)$. Then,

$$
f(T)-\lambda_{0}=\left(T-\lambda_{1}\right)^{k_{1}} \cdots\left(T-\lambda_{n}\right)^{k_{n}} g(T),
$$

where $g(T)$ is invertible.

Since $\lambda_{0} \in$ iso $\sigma_{a}(f(T))$, it follows from Lemma 2.6 that there exist $\delta_{i}>0$ such that $B_{\delta_{i}}\left(\lambda_{i}\right) \backslash\left\{\lambda_{i}\right\} \subset \mathbb{C} \backslash \sigma_{a}(T)(1 \leq i \leq n)$. Then, for each $i$, either $\lambda_{i} \notin \sigma_{a}(T)$ or $\lambda_{i} \in$ iso $\sigma_{a}(T)$. So, it remains to prove that $\lambda_{i} \in \rho_{s-F}(T)$ for all $i$. Now let $i$ be fixed and, without loss of generality, we assume that $\lambda_{i} \in$ iso $\sigma_{a}(T)$.

Since $0<\operatorname{nul}\left(f(T)-\lambda_{0}\right)<\infty$, there exists some $i_{0}\left(1 \leq i_{0} \leq n\right)$ such that $0<$ nul $\left(T-\lambda_{i_{0}}\right)<\infty$. Hence, $\lambda_{i_{0}} \in \pi_{00}^{a}(T)$. Since $T \in(\mathrm{a}-\mathrm{W})$, by Lemma 3.2, we have $\lambda_{i_{0}} \in \rho_{s-F}(T)$ and hence $\lambda_{i_{0}} \in \sigma_{p}(T) \cap\left[\rho_{s-F}^{-}(T) \cup \rho_{s-F}^{0}(T)\right]$. By condition (iii), we have $\lambda_{i} \in \sigma_{p}(T)$. Note that $0<\operatorname{nul}\left(\lambda_{i}-T\right) \leq \operatorname{nul}\left(\lambda_{0}-f(T)\right)<\infty$, then $\lambda_{i} \in \pi_{00}^{a}(T)$ and, using Lemma 3.2 again, we have $\lambda_{i} \in \rho_{s-F}(T)$. Thus, we conclude the proof. 
If one checks the proof of Main Theorem 1.1, then one can easily obtain the following result.

Corollary 3.4. Let $T \in \mathcal{B}(\mathcal{X})$. Then, $f(T) \in(\mathrm{a}-\mathrm{W})$ for all $f \in \operatorname{Hol}(\sigma(T))$ if and only if $p(T) \in(\mathrm{a}-\mathrm{W})$ for each polynomial $p(\lambda)$.

4. Proof of Main Theorem 1.2. We first give a useful lemma.

LemMa 4.1. Let $T \in \mathcal{B}(\mathcal{X})$. Then, $T \in(\omega)$ if and only if

(i) $\sigma(T)=\sigma_{w}(T) \cup \sigma_{0}(T)$,

(ii) $\pi_{00}(T) \subseteq \sigma_{0}(T)$, and

(iii) $\rho_{s-F}^{-}(T) \cap \sigma_{p}(T)=\emptyset$.

Proof. By Lemma 3.1, $T \in(\omega)$ if and only if $\left[\rho_{s-F}^{-}(T) \cup \rho_{s-F}^{0}(T)\right] \cap \sigma_{p}(T)=\pi_{00}(T)$.

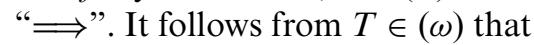

$$
\begin{aligned}
\pi_{00}(T) & =\left[\rho_{s-F}^{-}(T) \cup \rho_{s-F}^{0}(T)\right] \cap \sigma_{p}(T) \\
& =\left[\rho_{s-F}^{-}(T) \cap \sigma_{p}(T)\right] \cup\left[\rho_{s-F}^{0}(T) \cap \sigma_{p}(T)\right] .
\end{aligned}
$$

Since $\pi_{00}(T) \subseteq$ iso $\sigma(T)$ and $\rho_{s-F}^{-}(T) \subseteq$ int $\sigma(T)$, it follows that $\rho_{s-F}^{-}(T) \cap \sigma_{p}(T)=\emptyset$ and $\left[\rho_{s-F}^{0}(T) \cap \sigma_{p}(T)\right] \subseteq$ iso $\sigma(T)$. By Lemma 2.3, $\left[\rho_{s-F}^{0}(T) \cap \sigma_{p}(T)\right] \subseteq \sigma_{0}(T)$. Hence, $\sigma(T)=\sigma_{w}(T) \cup \sigma_{0}(T)$.

On the other hand, $\pi_{00}(T) \subseteq\left[\sigma_{a}(T) \backslash \sigma_{e a}(T)\right]$ implies that $\pi_{00}(T) \subseteq \rho_{s-F}(T)$. Then, by Lemma 2.3, we have $\pi_{00}(T) \subseteq \sigma_{0}(T)$.

" $\Longleftarrow$. By (i) and (iii), it is obvious that

$$
\begin{aligned}
\sigma_{a}(T) \backslash \sigma_{e a}(T) & =\left[\rho_{s-F}^{-}(T) \cup \rho_{s-F}^{0}(T)\right] \cap \sigma_{p}(T) \\
& =\left[\rho_{s-F}^{-}(T) \cap \sigma_{p}(T)\right] \cup\left[\rho_{s-F}^{0}(T) \cap \sigma_{p}(T)\right] \\
& =\rho_{s-F}^{0}(T) \cap \sigma_{p}(T)=\sigma_{0}(T) .
\end{aligned}
$$

Then, $\sigma_{a}(T) \backslash \sigma_{e a}(T)=\sigma_{0}(T) \subseteq \pi_{00}(T)$. This combining (ii) implies that $\sigma_{a}(T) \backslash$ $\sigma_{e a}(T)=\pi_{00}(T)$.

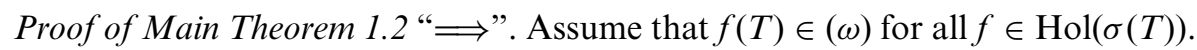

(i) Since $f(T) \in(\omega)$ for all $f \in \operatorname{Hol}(\sigma(T))$, we have $T=f_{1}(T) \in(\omega)$, where $f_{1}(\lambda)=\lambda$.

(ii) If (ii) does not hold, then we can choose $\lambda_{1} \in \rho_{s-F}^{-}(T)$ and $\lambda_{2} \in\left[\rho_{s-F}(T) \cap\right.$ $\left.\sigma_{p}(T)\right]$ such that $0 \leq$ ind $\left(T-\lambda_{2}\right)<\infty$. Obviously we can choose $k \in \mathbb{N}$ such that $k$. ind $\left(T-\lambda_{1}\right)+$ ind $\left(T-\lambda_{2}\right)<0$. Set $f_{2}(\lambda)=\left(\lambda-\lambda_{1}\right)^{k}\left(\lambda-\lambda_{2}\right)$. Then, $f_{2}(T)=(T-$ $\left.\lambda_{1}\right)^{k}\left(T-\lambda_{2}\right)$ is a semi-Fredholm operator and

$$
\text { ind } \begin{aligned}
f_{2}(T) & =\text { ind }\left(T-\lambda_{1}\right)^{k}\left(T-\lambda_{2}\right) \\
& =k \cdot \text { ind }\left(T-\lambda_{1}\right)+\text { ind }\left(T-\lambda_{2}\right)<0 .
\end{aligned}
$$

Evidently, $\operatorname{nul} f_{2}(T) \geq \operatorname{nul}\left(T-\lambda_{2}\right)>0$. Thus $0 \in\left[\rho_{s-F}^{-}\left(f_{2}(T)\right) \cap \sigma_{p}\left(f_{2}(T)\right)\right] \neq \emptyset$. By Lemma 4.1, we obtain $f_{2}(T) \notin(\omega)$, a contradiction.

(iii) If (iii) does not hold, then we can choose $\lambda_{1} \in \sigma_{0}(T)$ and $\lambda_{2} \in$ iso $\sigma(T)$ such that $\lambda_{2} \notin \sigma_{p}(T)$. Thus, nul $\left(T-\lambda_{2}\right)=0$ and, by Lemma 2.3 , we have $\lambda_{2} \in \sigma_{\text {lre }}(T)$.

Set $f_{3}(\lambda)=\left(\lambda-\lambda_{1}\right)\left(\lambda-\lambda_{2}\right)$. It is easy to verify that $0 \in \sigma_{l r e}\left(f_{3}(T)\right)$ and $0<$ nul $f_{3}(T)=\operatorname{nul}\left(T-\lambda_{1}\right)<\infty$. On the other hand, since $\lambda_{1}, \lambda_{2} \in$ iso $\sigma(T)$, by Corollary 
2.10, we have $0 \in$ iso $\sigma\left(f_{3}(T)\right)$. Thus, we obtain that $0 \in \pi_{00}\left(f_{3}(T)\right)$. Since $0 \in \sigma_{\text {lre }}\left(f_{3}(T)\right)$, by Lemma 4.1 , it follows that $f_{3}(T) \notin(\omega)$, a contradiction.

"£”. Arbitrarily choose an $f \in \operatorname{Hol}(\sigma(T))$. We shall prove that $f(T) \in(\omega)$.

Step 1. $\left[\sigma_{a}(f(T)) \backslash \sigma_{e a}(f(T))\right] \subseteq \pi_{00}(f(T))$.

Arbitrarily choose a $\lambda_{0} \in\left[\sigma_{a}(f(T)) \backslash \sigma_{e a}(f(T))\right]$. Then, by Lemma 3.1, $0<$ nul $\left(f(T)-\lambda_{0}\right)<\infty$ and ind $\left(\lambda_{0}-f(T)\right) \leq 0$. It suffices to prove that $\lambda_{0} \in$ iso $\sigma(f(T))$. By Lemma 2.6 and Lemma 2.8, we may assume that $\left\{\lambda_{i}\right\}_{i=1}^{n}$ is an enumeration of $\left\{z \in \sigma(T): f(z)-\lambda_{0}=0\right\}$ and

$$
f(z)-\lambda_{0}=\left(z-\lambda_{1}\right)^{k_{1}} \cdots\left(z-\lambda_{n}\right)^{k_{n}} g(z),
$$

where $g(z) \neq 0$ for all $z \in \sigma(T)$. Hence,

$$
f(T)-\lambda_{0}=\left(T-\lambda_{1}\right)^{k_{1}} \cdots\left(T-\lambda_{n}\right)^{k_{n}} g(T),
$$

where $g(T)$ is invertible.

It follows from ind $\left(f(T)-\lambda_{0}\right) \leq 0$ that $\lambda_{i} \in \rho_{s-F}(T)$ and ind $\left(\lambda_{i}-T\right)<\infty$ for all $i$. We claim that ind $\left(\lambda_{i}-T\right) \geq 0$ for all $1 \leq i \leq n$. In fact, if not, then there exists some $i_{0}$ such that ind $\left(T-\lambda_{i_{0}}\right)<0$. By condition (ii), $\sigma_{0}(T)=\emptyset$ and ind $\left(T-\lambda_{i}\right) \leq 0$ for all $i$. By Lemma 4.1, it follows from $T \in(\omega)$ that $\sigma(T)=\sigma_{w}(T)$, ind $\left(T-\lambda_{i}\right)<0$ and $T-\lambda_{i}$ is bounded below for all $1 \leq i \leq n$. Furthermore, $f(T)-\lambda_{0}$ is bounded below. Then, $\lambda_{0} \notin \sigma_{a}(f(T))$, a contradiction. Thus, we have proved that ind $\left(T-\lambda_{i}\right) \geq 0$ for all $1 \leq i \leq n$.

Since $\sum_{i=1}^{n} k_{i} \cdot$ ind $\left(T-\lambda_{i}\right)=$ ind $\left(\lambda_{0}-f(T)\right) \leq 0$, we deduce that ind $\left(T-\lambda_{i}\right)=0$ for all $i$. Note that $T \in(\omega)$ and $\lambda_{i} \in \sigma(T)$, it follows from Lemma 4.1 that $\lambda_{i} \in \sigma_{0}(T)$ for all $i$. In view of the form of $f(\lambda)$, it follows from Corollary 2.10 that $\lambda_{0} \in$ iso $\sigma(f(T))$.

Step 2. $\pi_{00}(f(T)) \subseteq\left[\sigma_{a}(f(T)) \backslash \sigma_{e a}(f(T))\right]$.

Arbitrarily choose a $\lambda_{0} \in \pi_{00}(f(T))$. Then, $0<\operatorname{nul}\left(f(T)-\lambda_{0}\right)<\infty$ and $\lambda_{0} \in$ iso $\sigma(f(T))$. By Lemma 2.4 and Lemma 3.1, it suffices to prove that $\lambda_{0} \in \rho_{s-F}(f(T))$. Note that nul $\left(f(T)-\lambda_{0}\right)<\infty$, then, by Corollary 2.7 and Lemma 2.8 , we may assume that $\left\{\lambda_{i}\right\}_{i=1}^{n}$ is an enumeration of $\left\{\lambda \in \sigma(T): f(\lambda)-\lambda_{0}=0\right\}$ and

$$
f(z)-\lambda_{0}=\left(z-\lambda_{1}\right)^{k_{1}} \cdots\left(z-\lambda_{n}\right)^{k_{n}} g(z),
$$

where $g(z) \neq 0$ for all $z \in \sigma(T)$ and $\lambda_{i} \in$ iso $\sigma(T)$ for all $i$. Then,

$$
f(T)-\lambda_{0}=\left(T-\lambda_{1}\right)^{k_{1}} \cdots\left(T-\lambda_{n}\right)^{k_{n}} g(T),
$$

where $g(T)$ is invertible.

Since $0<\operatorname{nul}\left(f(T)-\lambda_{0}\right)<\infty$, there exists some $i_{0}\left(1 \leq i_{0} \leq n\right)$ such that $0<$ nul $\left(T-\lambda_{i_{0}}\right)<\infty$. Hence, $\lambda_{i_{0}} \in \pi_{00}(T)$. Since $T \in(\omega)$, by Lemma 4.1 , we have $\lambda_{i_{0}} \in \sigma_{0}(T)$. So, $\sigma_{0}(T) \neq \varnothing$ and, by condition (iii), iso $\sigma(T) \subseteq \sigma_{p}(T)$. It follows that $\lambda_{i} \in \sigma_{p}(T)$ for all $i$. Note that nul $\left(T-\lambda_{i}\right) \leq \operatorname{nul}\left(f(T)-\lambda_{0}\right)<\infty$ for all $i$, we deduce that $\lambda_{i} \in \pi_{00}(T)$. Using Lemma 4.1 again, we obtain $\lambda_{i} \in \sigma_{0}(T)$ for all $i$. Therefore, we conclude that $\lambda_{0} \in \rho_{s-F}(f(T))$.

It can be seen from the proof of Main Theorem 1.2 that the following corollary is clear.

COROLlary 4.2. Let $T \in \mathcal{B}(\mathcal{X})$. Then, $f(T) \in(\omega)$ for all $f \in \operatorname{Hol}(\sigma(T))$ if and only if $p(T) \in(\omega)$ for each polynomial $p(\lambda)$. 
5. Proof of Main Theorem 1.3. In this section, it is always assumed that $\mathcal{X}$ is a complex separable infinite dimensional Hilbert space. We first give several useful lemmas.

Lemma 5.1 ([15], Theorem 3.48). Let $T \in \mathcal{B}(\mathcal{X})$. Then, given $\varepsilon>0$, there exists $K \in \mathcal{K}(\mathcal{X})$ such that $\|K\|<\varepsilon+\max \left\{\operatorname{dist}\left[\lambda, \partial \rho_{s-F}(T)\right]: \lambda \in \sigma_{0}(T)\right\}$ and $\min$ ind $(T+$ $K-\lambda)=0$ for all $\lambda \in \rho_{s-F}(T)$.

For $T \in \mathcal{B}(\mathcal{X})$ and $\lambda \in \rho_{s-F}(T)$, the minimal index (see [15]) of $\lambda-T$ is defined by

$$
\min \text { ind }(\lambda-T):=\min \left\{\operatorname{nul}(\lambda-T), \operatorname{nul}(\lambda-T)^{*}\right\} .
$$

Lemma 5.2 ([16], Proposition 3.4). Let $T \in \mathcal{B}(\mathcal{X})$. If $\sigma_{0}(T)=\emptyset$, then, given $\varepsilon>0$, there exists $K \in \mathcal{K}(\mathcal{X})$ with $\|K\|<\varepsilon$ such that $\sigma_{p}(T+K)=\rho_{s-F}^{+}(T)$.

Proof of Main Theorem 1.3. For given $\varepsilon>0$, set $\sigma_{1}=\left\{\lambda \in \sigma_{0}(T)\right.$ : $\left.\operatorname{dist}\left(\lambda, \partial \rho_{s-F}(T)\right) \geq \frac{\varepsilon}{2}\right\}$. Then, $\sigma_{1}$ is a finite, clopen subset of $\sigma(T)$. Set $\sigma_{2}=\sigma(T) \backslash \sigma_{1}$. By Corollary 2.2, $T$ admits the following representation

$$
T=\left[\begin{array}{cc}
T_{1} & E \\
0 & T_{2}
\end{array}\right] \begin{gathered}
\mathcal{X}\left(\sigma_{1} ; T\right) \\
\mathcal{X}\left(\sigma_{1} ; T\right)^{\perp}
\end{gathered}
$$

where $\sigma\left(T_{i}\right)=\sigma_{i}(i=1,2)$. Then, one can verify that $\max \left\{\operatorname{dist}\left[\lambda, \partial \rho_{s-F}\left(T_{2}\right)\right]: \lambda \in\right.$ $\left.\sigma_{0}\left(T_{2}\right)\right\}<\frac{\varepsilon}{2}$. Then, by Lemma 5.1, there exists a compact operator $K_{1}$ on $\mathcal{X}\left(\sigma_{1} ; T\right)^{\perp}$ such that $\left\|K_{1}\right\|<\frac{\varepsilon}{2}$ and min ind $\left(T_{2}+K_{1}-\lambda\right)=0$ for all $\lambda \in \rho_{s-F}\left(T_{2}\right)$. Then,

$$
\begin{aligned}
\sigma\left(T_{2}+K_{1}\right) & =\sigma_{\text {lre }}\left(T_{2}+K_{1}\right) \cup\left[\rho_{s-F}\left(T_{2}+K_{1}\right) \cap \sigma\left(T_{2}+K_{1}\right)\right] \\
& =\sigma_{\text {lre }}\left(T_{2}\right) \cup \rho_{s-F}^{+}\left(T_{2}+K_{1}\right) \cup \rho_{s-F}^{-}\left(T_{2}+K_{1}\right) \cup\left[\rho_{s-F}^{0}\left(T_{2}+K_{1}\right) \cap \sigma\left(T_{2}+K_{1}\right)\right] \\
& =\sigma_{\text {lre }}\left(T_{2}\right) \cup \rho_{s-F}^{+}\left(T_{2}\right) \cup \rho_{s-F}^{-}\left(T_{2}\right) \subset \sigma\left(T_{2}\right) .
\end{aligned}
$$

In particular, $\sigma_{0}\left(T_{2}+K_{1}\right)=\emptyset$ and $\sigma\left(T_{2}+K_{1}\right) \cap \sigma\left(T_{1}\right)=\emptyset$.

Using Lemma 5.2, one can find a compact $K_{2}$ with $\left\|K_{2}\right\|<\varepsilon / 2$ such that $\sigma_{p}\left(T_{2}+\right.$ $\left.K_{1}+K_{2}\right)=\rho_{s-F}^{+}\left(T_{2}+K_{1}+K_{2}\right)$. Set

$$
\overline{T_{2}}=T_{2}+K_{1}+K_{2} \quad \text { and } \quad K=\left[\begin{array}{cc}
0 & 0 \\
0 & K_{1}+K_{2}
\end{array}\right] \begin{gathered}
\mathcal{X}\left(\sigma_{1} ; T\right) \\
\mathcal{X}\left(\sigma_{1} ; T\right)^{\perp}
\end{gathered}
$$

Then, $K \in \mathcal{K}(\mathcal{X}),\|K\|<\varepsilon$ and

$$
T+K=\left[\begin{array}{cc}
T_{1} & E \\
0 & T_{2}
\end{array}\right] \begin{gathered}
\mathcal{X}\left(\sigma_{1} ; T\right) \\
\mathcal{X}\left(\sigma_{1} ; T\right)^{\perp}
\end{gathered}
$$

Also, we claim that

(i) $\sigma(T+K)=\sigma\left(T_{1}\right) \cup \sigma\left(\overline{T_{2}}\right)$ and $\sigma\left(T_{1}\right) \cap \sigma\left(\overline{T_{2}}\right)=\emptyset$,

(ii) $\sigma_{\text {lre }}(T+K)=\sigma_{\text {lre }}\left(\overline{T_{2}}\right)$ and ind $(T+K-\lambda)=\operatorname{ind}\left(\overline{T_{2}}-\lambda\right)$ for all $\lambda \in \rho_{s-F}(T+$ $K)$,

(iii) $\sigma_{p}(T+K)=\sigma_{p}\left(T_{1}\right) \cup \sigma_{p}\left(\overline{T_{2}}\right)=\sigma\left(T_{1}\right) \cup \rho_{s-F}^{+}\left(\overline{T_{2}}\right)=\sigma\left(T_{1}\right) \cup \rho_{s-F}^{+}(T+K)$,

(iv) $\sigma_{0}(T+K)=\sigma\left(T_{1}\right)=\pi_{00}^{a}(T)=\pi_{00}(T)\left(\right.$ since $\left.\sigma_{p}\left(\overline{T_{2}}\right)=\rho_{s-F}^{+}\left(\overline{T_{2}}\right)\right)$.

Now let us explain in detail the above facts (i)-(iv).

(i) Using a similar argument as in the equality (1), one can see that $\sigma\left(T_{1}\right) \cap \sigma\left(\overline{T_{2}}\right)=$ $\emptyset$. By [15, Corollary 3.22], $T+K$ and $T_{1} \oplus \overline{T_{2}}$ are similar. Then, $\sigma(T+K)=\sigma\left(T_{1}\right) \cup$ $\sigma\left(\overline{T_{2}}\right)$. 
(ii) Since $\operatorname{dim} \mathcal{X}\left(\sigma_{1} ; T\right)<\infty$, one can see that $T_{1}$ and $E$ are both compact. Thus, $T+K$ is a compact perturbation of the following operator

$$
\left[\begin{array}{cc}
0 & 0 \\
0 & \overline{T_{2}}
\end{array}\right] \begin{gathered}
\mathcal{X}\left(\sigma_{1} ; T\right) \\
\mathcal{X}\left(\sigma_{1} ; T\right)^{\perp}
\end{gathered}
$$

Thus, the facts in (ii) are clear.

(iii) We have proved in (i) that $T+K$ and $T_{1} \oplus \overline{T_{2}}$ are similar. Then, $\sigma_{p}(T+K)=$ $\sigma_{p}\left(T_{1}\right) \cup \sigma_{p}\left(\overline{T_{2}}\right)$. Note that $T_{1}$ is acting on a finite-dimensional space, we have $\sigma\left(T_{1}\right)=$ $\sigma_{p}\left(T_{1}\right)$. On the other hand, we have proved that $\sigma_{p}\left(\overline{T_{2}}\right)=\rho_{s-F}^{+}\left(\overline{T_{2}}\right)=\rho_{s-F}^{+}(T+K)$. This proves (iii).

(iv) Since $T+K$ and $T_{1} \oplus \overline{T_{2}}$ are similar, using the facts (i)-(iii), one can easily verify the conditions in (iv).

Based on the facts (i)-(iv), we obtain

$$
\begin{aligned}
& {\left[\rho_{s-F}^{-}(T+K) \cup \rho_{s-F}^{0}(T+K)\right] \cap \sigma_{p}(T+K)} \\
& \quad=\rho_{s-F}^{0}(T+K) \cap \sigma_{p}(T+K) \\
& \quad=\sigma_{0}(T+K)=\sigma\left(T_{1}\right)=\pi_{00}^{a}(T)=\pi_{00}(T) .
\end{aligned}
$$

By Lemma 3.1, we deduce that $T+K \in(\mathrm{a}-\mathrm{W})$ and $T+K \in(\omega)$.

REMARK 5.3. As we have seen in the above proof, the result of Theorem 1.3 greatly depends on the work by D. Herrero [16] on perturbations of Hilbert space operators, and therefore, the result is established only in the setting of separable Hilbert spaces.

We conclude this paper with the following question.

Question 5.4. Let $\mathcal{X}$ be a complex infinite dimensional Banach space. Then, given $T \in \mathcal{B}(\mathcal{X})$ and $\varepsilon>0$, can one find $A \in \mathcal{B}(\mathcal{X})$ with $\|A-T\|<\varepsilon$ such that $A \in(\mathrm{W})$ (or $A \in(\omega)$, or $A \in(\mathrm{a}-\mathrm{W}))$ ?

\section{REFERENCES}

1. P. Aiena, Fredholm and local spectral theory, with applications to multipliers (Kluwer Academic Publishers, Dordrecht, 2004). $105-122$

3. P. Aiena, Property (w) and perturbations. II, J. Math. Anal. Appl. 342(2) (2008), 830837.

4. P. Aiena, M. T. Biondi and F. Villafañe, Property $(w)$ and perturbations. III, J. Math. Anal. Appl. 353(1) (2009), 205-214.

5. P. Aiena and P. Peña, Variations on Weyl's theorem, J. Math. Anal. Appl. 324 (1) (2006),
566-579.

6. I. J. An and Y. M. Han, Weyl's theorem for algebraically quasi-class A operators, Int. Equ. Oper. Theory 62 (1) (2008), 1-10. $187-196$

7. B. A. Barnes, Riesz points and Weyl's theorem, Int. Equ. Oper. Theory 34(2) (1999),

8. M. Chō and Y. M. Han, Riesz idempotent and algebraically $M$-hyponormal operators, Int. Equ. Oper. Theory 53(3) (2005), 311-320. $285-288$

9. L. A. Coburn, Weyl's theorem for nonnormal operators, Michigan Math. J. 13 (1966),

10. J. B. Conway, A course in functional analysis, second ed. Graduate Texts in Mathematics, vol. 96, (Springer-Verlag, New York, 1990). 
11. R. E. Curto and Y. M. Han, Weyl's theorem, $a$-Weyl's theorem, and local spectral theory, J. London Math. Soc. (2) 67(2) (2003), 499-509.

12. R. E. Curto and Y. M. Han, Weyl's theorem for algebraically paranormal operators, Int. Equ. Oper. Theory 47 (3) (2003), 307-314.

13. B. P. Duggal, The Weyl spectrum of $p$-hyponormal operators, Int. Equ. Oper. Theory 29(2) (1997)197-201.

14. Y. M. Han and W. Y. Lee, Weyl's theorem holds for algebraically hyponormal operators, Proc. Amer. Math. Soc. 128 (8) (2000), 2291-2296 (electronic).

15. D. A. Herrero, Approximation of Hilbert space operators, vol. 1, second ed., Pitman Research Notes in Mathematics Series, vol. 224 (Longman Scientific \& Technical, Harlow, 1989).

16. D. A. Herrero, T. J. Taylor and Z. Y. Wang, Variation of the point spectrum under compact perturbations, in Topics in operator theory, Vol. 32 of Oper. Theory Adv. Appl., Birkhäuser, Basel, 1988, pp. 113-158.

17. C. G. Li, S. Zhu and Y. L. Feng, Weyl's theorem for functions of operators and approximation, Int. Equ. Oper. Theory 67(4) (2010), 481-497.

18. M. Oudghiri, Weyl's and Browder's theorems for operators satisfying the SVEP, Studia Math. 163(1) (2004), 85-101.

19. H. Radjavi and P. Rosenthal, Invariant subspaces, second ed. (Dover Publications, Mineola, NY, 2003).

20. V. Rakočević, On one subset of M. Schechter's essential spectrum, Mat. Vesnik 5(18) (33)(4) (1981), 389-391.

21. V. Rakočević, On the essential approximate point spectrum. II, Mat. Vesnik 36(1) (1984), 89-97.

22. V. Rakočević, On a class of operators, Mat. Vesnik 37(4) (1985), 423-426.

23. V. Rakočević, Approximate point spectrum and commuting compact perturbations, Glasgow Math. J. 28(2) (1986), 193-198. 